\title{
Development of microwave diagnostic simulator
}

\author{
H. Hojo, Y. Kurosawa, and A. Mase \\ Plasma Research Center, University of Tsukuba, Tsukuba 305-8577, Japan
}

(Presented on 10 June 1998)

Two-dimensional simulator models are presented for microwave diagnostics. The models assume the Maxwell wave equation coupled with the equation of plasma current density in a cold magnetized plasma, which can describe propagation, reflection, and cross polarization scattering of the ordinary and extraordinary modes. The effects of waveguide for the microwave launcher and wall boundary of the vacuum vessel are included in the models. The simulations of ultrashort-pulse reflectometry with the use of incident subcyclic ordinary modes in the models are performed to test the problem of density profile reconstruction. (c) 1999 American Institute of Physics.

[S0034-6748(99)67301-4]

\section{INTRODUCTION}

Microwave diagnostics such as reflectometry ${ }^{1-4}$ and cross polarization scattering ${ }^{5-7}$ are recently receiving growing attention in magnetic confinement fusion research. In order to obtain a better understanding of plasma confinement physics, more detailed measurements on plasma profiles and fluctuations might be required. Recently, Domier et al. ${ }^{8}$ have proposed a new type of microwave reflectometry (called ultrashort-pulse reflectometry) with the use of subcyclic microwave pulses, expected as a near-coming diagnostic. A subcyclic pulse can be considered as a set of many monochromatic plane waves with different frequencies which have different cutoff densities, therefore the ultrashort-pulse reflectometry has the potential to measure precisely the profiles and fluctuations with respect to plasma density and magnetic field by only a single pulse. The theoretical studies on this ultrashort-pulse reflectometry have also been done by Cohen et al. ${ }^{9-11}$ and Hojo et al. ${ }^{6,12}$

In this article, we present two-dimensional simulator models to investigate microwave diagnostics such as reflectometry and cross polarization scattering in magnetically confined plasmas. Using these models, we perform the simulations of ultrashort-pulse reflectometry with the use of subcyclic ordinary modes, and then test the problem of density profile reconstruction in these models. In the Sec. II we describe the simulator modeling, and in Sec. III we show the simulation results on ultrashort-pulse reflectometry in these models. The summary is presented in Sec. IV.

\section{SIMULATOR MODELING}

In this section, we describe two-dimensional simulator models for microwave diagnostics. Two models (the XZ and $\mathrm{XY}$ models) shown in Fig. 1 are considered, and both models roughly image the central cell of the tandem mirror GAMMA 10 at the University of Tsukuba. The basic equations to be solved are the Maxwell wave equation for the electric field $\mathbf{E}$ and the equation for the current density $\mathbf{J}$ as follows:

$$
\begin{aligned}
& \frac{\partial^{2}}{\partial t^{2}} \mathbf{E}+c^{2} \nabla(\nabla \cdot \mathbf{E})-c^{2} \nabla^{2} \mathbf{E}+\frac{1}{\epsilon_{0}} \frac{\partial}{\partial t} \mathbf{J}=0, \\
& \frac{1}{\epsilon_{0}} \frac{\partial}{\partial t} \mathbf{J}=\omega_{\mathrm{pe}}^{2} \mathbf{E}-\frac{e}{m_{e} \epsilon_{0}} \mathbf{J} \times \mathbf{B}_{0},
\end{aligned}
$$

where $c$ is the speed of light, $\omega_{\text {pe }}\left(=\sqrt{e^{2} n / m_{e} \epsilon_{0}}\right)$ the electron plasma frequency, $-e$ the charge of the electron, $m_{e}$ the electron mass, $n$ the plasma density, $\epsilon_{0}$ the permittivity of vacuum, and $\mathbf{B}_{0}$ the external magnetic field. In the derivation of Eq. (2), we assumed that the current density is approximated as $\mathbf{J}=-e n v_{e}, v_{e}$ being the electron flow velocity, as we consider electromagnetic waves in $\mathrm{GHz}$ range. The above coupled equation can describe the ordinary $(\mathrm{O})$ and extraordinary (X) modes. When $\mathbf{B}_{0}=B_{0} \hat{\mathbf{z}}, \hat{\mathbf{z}}$ being the unit vector in the $z$ direction, $E_{z}$ denotes the $O$ mode, and $E_{x}$ and $E_{y}$ correspond to the $\mathrm{X}$ mode. We assume conducting walls for the vacuum vessel and waveguide as the boundary condition of
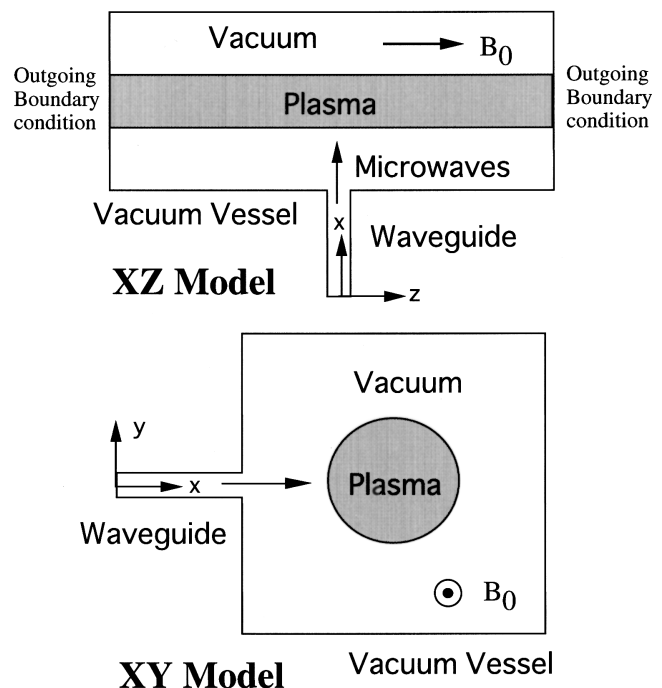

FIG. 1. Two-dimensional simulator models (XZ and XY). In the simulations of ultrashort-pulse reflectometry, the uniform magnetic field $\mathbf{B}_{0}$ is in the $z$ direction, and the density profile is Gaussian in the $x$ direction (XZ model) and in the $x$ and $y$ directions (XY model). 

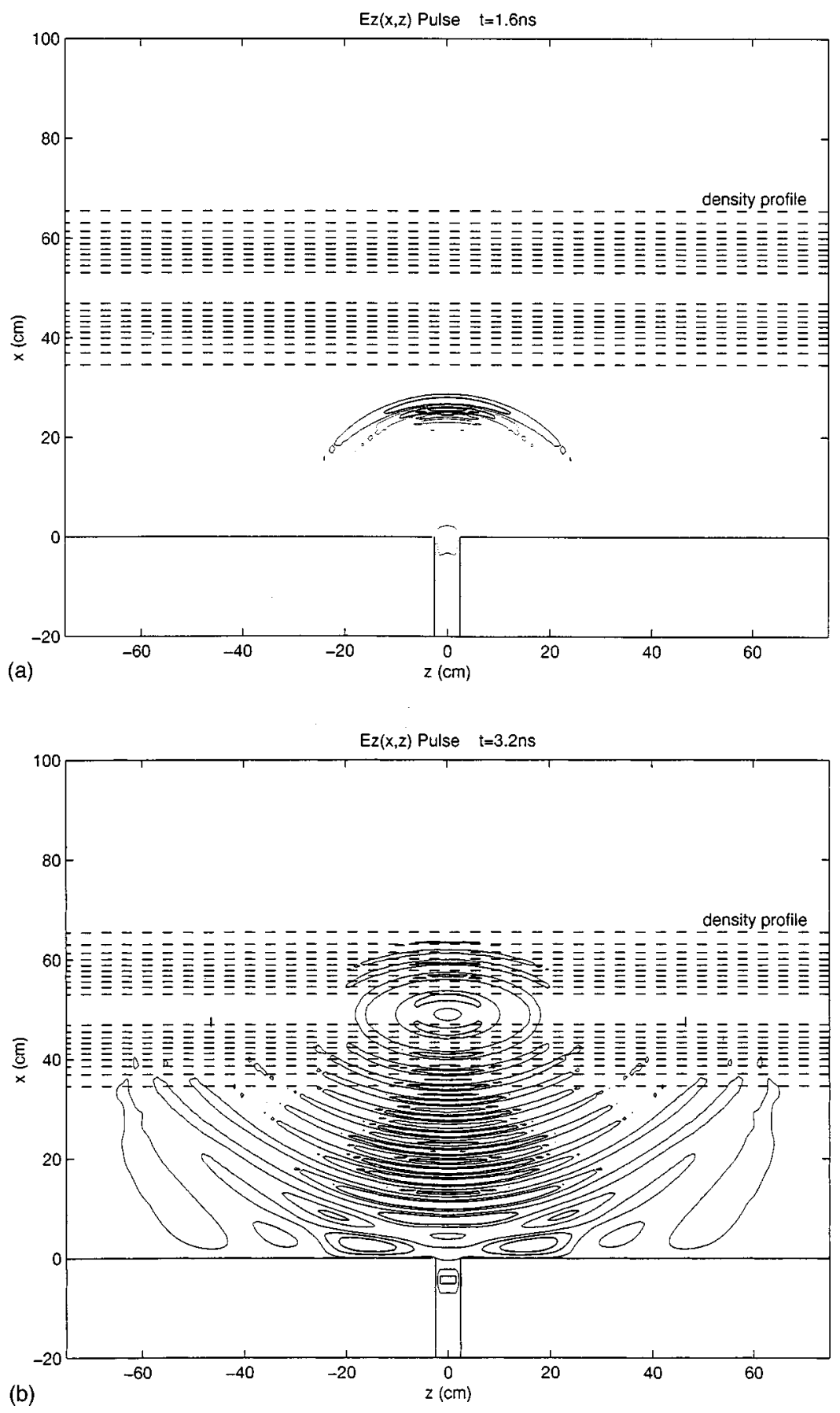

FIG. 2. Wave patterns of the electric field $E_{z}(x, z, t)$ at $t=1.6 \mathrm{~ns}$ (a) and $3.2 \mathrm{~ns}$ (b) in the $\mathrm{XZ}$ model. The dashed lines show the contour of the density profile.
Eq. (1), and also assume the boundary condition that the electric field $\mathbf{E}$ is outgoing on both sides of the vacuum vessel in the $\mathrm{XZ}$ model. In the present $\mathrm{XZ}$ model, the outgoing condition for the wave electric field is satisfied by the institution of wave absorbing layers on both sides of the vacuum vessel.

As the electromagnetic waves are in the $\mathrm{GHz}$ range, any fluctuations in $\mathrm{MHz}$ or the lower-frequency range can be treated to be static. If we intend to include such frozen fluctuations of the plasma density and magnetic field into Eqs. (1) and (2), we have only to replace $n$ and $\mathbf{B}_{0}$ in Eqs. (1) and (2) by $n+\delta n$ and $\mathbf{B}_{0}+\delta \mathbf{B}$, respectively, where $\delta n$ and $\delta \mathbf{B}$ denote the frozen fluctuations. In this case, we see that the Lorentz force term $\mathbf{J} \times \delta \mathbf{B}$ in Eq. (2) drives the cross polar- ization scattering between the $\mathrm{O}$ and $\mathrm{X}$ modes due to magnetic fluctuations. ${ }^{6}$

\section{ULTRASHORT-PULSE REFLECTOMETRY}

In this section, we study ultrashort-pulse reflectometry with use of the $\mathrm{O}$ mode pulse. ${ }^{8-13}$ The electric field of the incident $\mathrm{O}$-mode pulse in a waveguide obeys a wave equation, for example, in the XY model, as follows:

$$
\left[\frac{\partial^{2}}{\partial t^{2}}-c^{2}\left(\frac{\partial^{2}}{\partial x^{2}}+\frac{\partial^{2}}{\partial y^{2}}\right)\right] E_{z}(x, y, t)=0 .
$$

The subcyclic pulse solution of Eq. (3) for the $\mathrm{O}$ mode is given by 


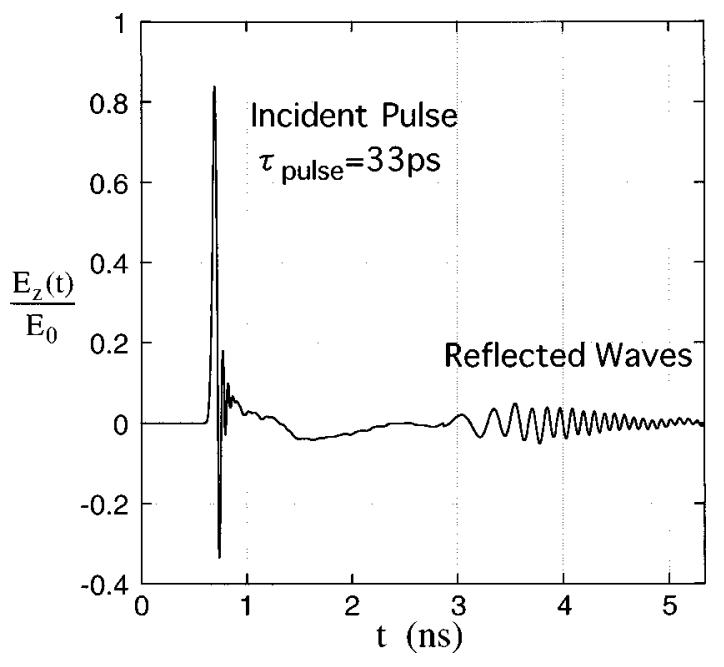

FIG. 3. The temporal evolution of the electric field $E_{z}(t)$ at $x=z=0$ in the XZ model.

$$
E_{z}(x, y, t)=E_{0} \cos (k y) F(x, t)
$$

with $A(t)=\exp \left[-(t / a)^{2}\right]$ (here, the half pulse width is given by $\tau_{p}=2 a \sqrt{\ln 2}$ ) and

$$
\begin{aligned}
F(x, t)= & \frac{1}{2 c} \frac{\partial}{\partial t} \int_{x-c t}^{x+c t} J_{0}\left[k \sqrt{(c t)^{2}-(\eta-x)^{2}}\right] A(\eta) d \eta \\
& -\frac{1}{2} \int_{x-c t}^{x+c t} J_{0}\left[k \sqrt{(c t)^{2}-(\eta-x)^{2}}\right] \frac{\partial A}{\partial \eta} d \eta,
\end{aligned}
$$

where $(\partial / \partial t+c \partial / \partial x) F(x, t)=0$ is assumed as the initial condition at $t=0$. Hereafter, we assume the incident $\mathrm{O}$-mode pulse with the pulse width $\tau_{p}=33 \mathrm{ps}$ and $k=\pi / 5 \mathrm{~cm}^{-1}$, where the width of waveguide is set to $5 \mathrm{~cm}$. The waveguide modes in the XZ model can be obtained in a way similar to Eqs. (3) through (5). We also assume the equilibrium density profile as $n(x)=n_{0} \exp \left\{-\left[\left(x-x_{0}\right) / L\right]^{2}\right\}$ in the XZ model, and

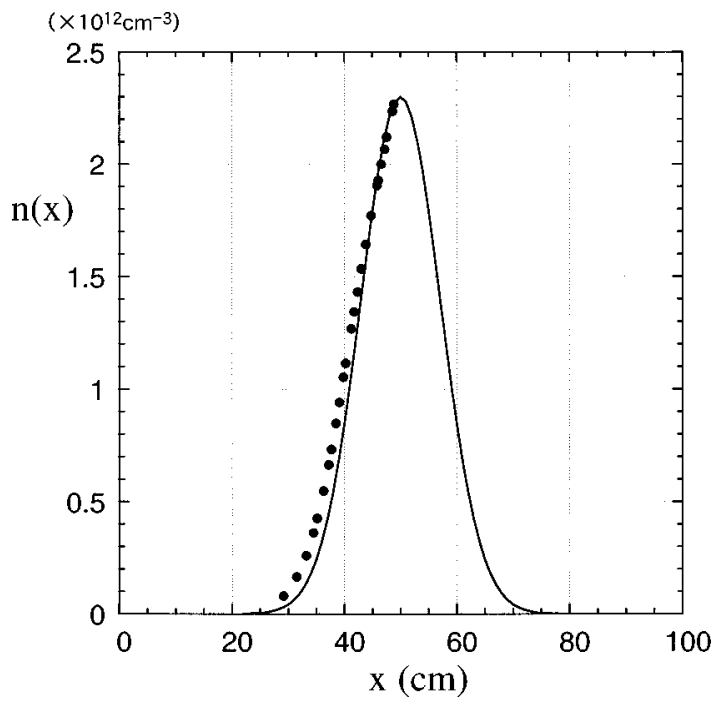

FIG. 4. The reconstructed (solid circles) and the original (solid line) density profiles in the $\mathrm{XZ}$ model.

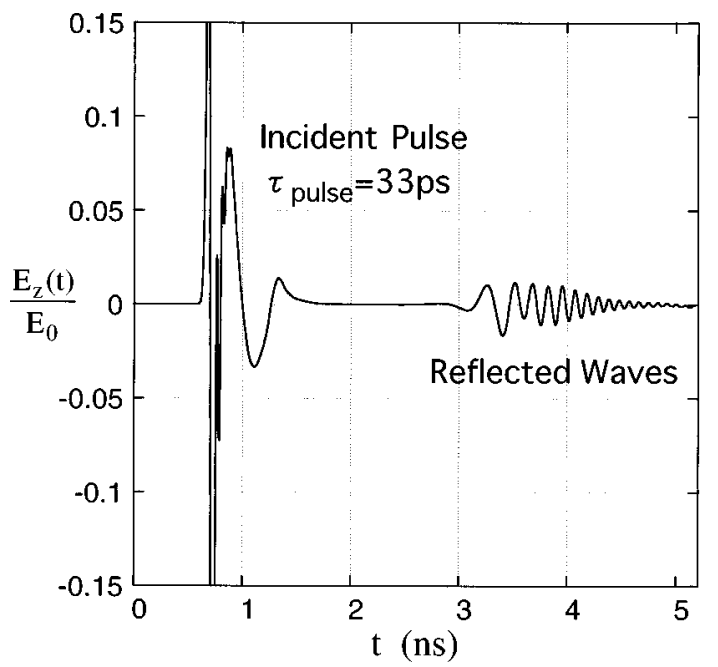

FIG. 5. The temporal evolution of the electric field $E_{z}(t)$ at $x=y=0$ in the XY model.

$n(x, y)=n_{0} \exp \left\{-\left[\left(x-x_{0}\right) / L\right]^{2}-\left[\left(y-y_{0}\right) / L\right]^{2}\right\} \quad$ in the $\mathrm{XY}$ model, where $L=10 \mathrm{~cm}, x_{0}=y_{0}=50 \mathrm{~cm}$, and $n_{0}=2.3$ $\times 10^{12} \mathrm{~cm}^{-3}$.

We now analyze the density profile reconstruction by the measurement of the $\mathrm{O}$ modes reflected at the cutoff. Following Ref. 9, the time delay $\tau(\omega)$ for a reflected wave with a frequency $\omega$ observed just in front of the wave guide (i.e., at $x=z=0$ in the XZ model and at $x=y=0$ in the XY model) is given by

$$
\tau(\omega)=\int_{0}^{x_{r}} d x \frac{2}{v_{g}}=\int_{0}^{x_{r}} d x \frac{2}{c} \frac{\omega}{\sqrt{\omega^{2}-\omega_{\mathrm{pe}}^{2}(x)}},
$$

where $x_{r}$ is the reflection point which is determined by the cutoff condition $\omega=\omega_{\text {pe }}(x)$ and $v_{g}$ is the group velocity of the $\mathrm{O}$ mode. From the Abel inversion of Eq. (6), we obtain, for the reflection point $x_{r}$,

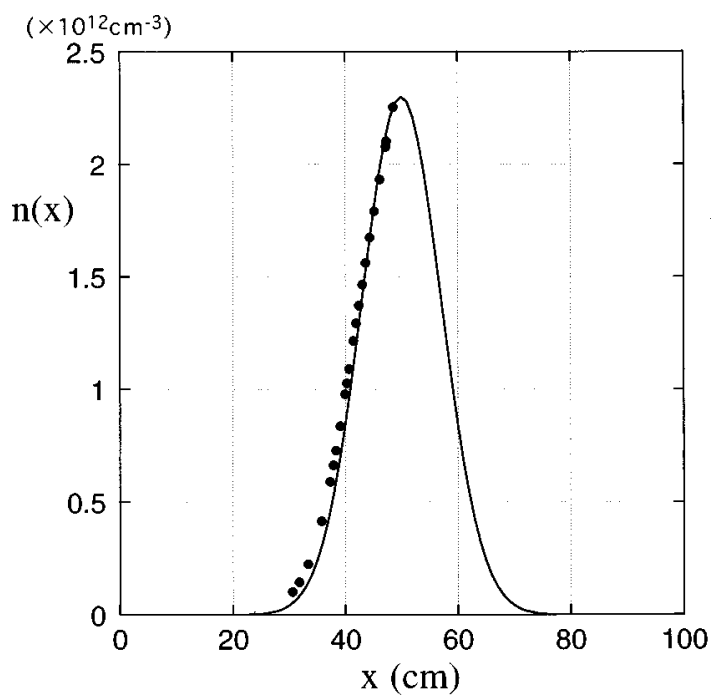

FIG. 6. The reconstructed (solid circles) and the original (solid line) density profiles in the XY model. 


$$
x_{r}\left(\omega_{\mathrm{pe}}\right)=\int_{0}^{\omega_{\mathrm{pe}}} d \omega \frac{c \tau(\omega)}{\pi \sqrt{\omega_{\mathrm{pe}}^{2}-\omega^{2}}} .
$$

Then we can reconstruct the density profile $n(x)$ by measuring the time delay $\tau(\omega)$ of the reflected wave with the frequency $\omega$. The time delay $\tau(\omega)$ of the reflected wave is computed by use of the peak counting method ${ }^{12}$ which is similar to the zero crossing method discussed in Ref. 9.

In Fig. 2, we show the wave patterns of the electric field $E_{z}(x, z, t)$ of the O mode at $t=1.6 \mathrm{~ns}$ (a) and $t=3.2 \mathrm{~ns}$ (b) in the XZ model. The dashed lines show the contour of the density profile. In Fig. 3, we show the temporal behavior of the electric wave field $E_{z}(t) / E_{0}$ of the $\mathrm{O}$ mode at $x=z=0$ in the $\mathrm{XZ}$ model. In the figure, the earlier signal shows the incident pulse and the later signal denotes the waves reflected at the cutoff. From these reflected wave signals, we obtain the time delay $\tau(\omega)$, and then by using Eq. (7) we can reconstruct the density profile $n(x)$. The reconstructed profile in the XZ model is shown in Fig. 4 by closed circles, where the solid line denotes the original density profile. We see that the reconstructed density profile coincides well with the original one.

In Figs. 5 and 6, we show the temporal behavior of the electric wave field $E_{z}(t) / E_{0}$ of the $\mathrm{O}$ mode at $x=y=0$ and the result of the density profile reconstruction, respectively, in the XY model. In this case, we also see that the reconstructed density profile coincides well with the original one.

\section{SUMMARY}

We presented here two-dimensional simulator models to investigate microwave diagnostics in magnetically confined plasmas. Using these models, we performed the simulations on ultrashort-pulse reflectometry with the use of subcyclic O-mode pulses, and then analyzed the density profile reconstruction. We found that the reconstructed density profile coincides well with the original one in both $\mathrm{XZ}$ and $\mathrm{XY}$ models.
The simulations of magnetic field reconstruction by using $\mathrm{X}$-mode reflectometry and cross polarization scattering diagnostics based on these models will be our next task.

\section{ACKNOWLEDGMENTS}

The authors are grateful to members of the Plasma Research Center, University of Tsukuba, for valuable discussions. This work was partly supported by a Grant-in-Aid for Scientific Research from the Ministry of Education, Science, Sports and Culture of Japan.

${ }^{1}$ A. Mase, M. Ichimura, H. Satake, R. Katsumata, T. Tokuzawa, Y. Ito, H. Hojo, E. J. Doyle, A. Itakura, M. Inutake, and T. Tamano, Phys. Fluids B 5, 1677 (1993).

${ }^{2}$ H. Hojo, A. Mase, M. Inutake, and M. Ichimura, J. Plasma Fusion Res. 69, 1043 (1993).

${ }^{3}$ A. Mase, H. Hojo, M. Kobayashi, N. Oyama, L. G. Bruskin, E. J. Doyle, T. Tokuzawa, A. Itakura, M. Ichimura, and T. Tamano, Diagnostics for Experimental Thermonuclear Fusion Reactors, edited by P. Stott, G. Gorini, and E. Sindoni (Plenum, New York, 1996), p. 153.

${ }^{4}$ C. Laviron, P. Millot, and R. Prentice, Plasma Phys. Controlled Fusion 37, 975 (1995).

${ }^{5}$ X. L. Zou, L. Colas, M. Paume, J. M. Chareau, L. Laurent, P. Devynck, and D. Gresillon, Phys. Rev. Lett. 75, 1090 (1995).

${ }^{6}$ N. Katsuragawa, H. Hojo, and A. Mase, J. Phys. Soc. Jpn. 67, 2574 (1998)

${ }^{7}$ Y. Kogi, A. Mase, L. G. Bruskin, N. Oyama, T. Tokuzawa, A. Itakura, H. Hojo, and T. Tamano, Jpn. J. Appl. Phys., Part 2 36, L587 (1997).

${ }^{8}$ C. W. Domier, N. C. Luhmann, Jr., A. E. Chou, W. M. Zhang, and A. J. Romanowski, Rev. Sci. Instrum. 66, 399 (1995).

${ }^{9}$ B. I. Cohen, B. B. Afeyan, A. E. Chou, and N. C. Luhmann, Jr., Plasma Phys. Controlled Fusion 37, 329 (1995).

${ }^{10}$ B. I. Cohen, T. B. Kaiser, and J. C. Garrison, Rev. Sci. Instrum. 68, 1238 (1997).

${ }^{11}$ B. I. Cohen, L. L. LoDestro, E. B. Hooper, and T. A. Casper, Plasma Phys. Controlled Fusion 40, 75 (1998).

${ }^{12}$ H. Hojo, H. Honda, N. Katsuragawa, and A. Mase, Fusion Eng. Des. 34-35, 447 (1997).

${ }^{13}$ S. Kubota, T. Onuma, A. Mase, T. Tokuzawa, N. Oyama, A. Itakura, H. Hojo, L. Bruskin, T. Tamano, K. Yatsu, C. Domier, and N. Luhmann, Jr., Jpn. J. Appl. Phys., Part 2 37, L300 (1998). 